\title{
Identification and characterization of EBV genomes in spontaneously immortalized human peripheral blood B lymphocytes by NGS technology
}

Haiyan Lei, Tianwei Li, Guo-Chiuan Hung, Bingjie Li, Shien Tsai and Shyh-Ching Lo*

\begin{abstract}
Background: We conducted genomic sequencing to identify Epstein Barr Virus (EBV) genomes in 2 human peripheral blood B lymphocytes that underwent spontaneous immortalization promoted by mycoplasma infections in culture, using the high-throughput sequencing (HTS) Illumina MiSeq platform. The purpose of this study was to examine if rapid detection and characterization of a viral agent could be effectively achieved by HTS using a platform that has become readily available in general biology laboratories.

Results: Raw read sequences, averaging 175 bps in length, were mapped with DNA databases of human, bacteria, fungi and virus genomes using the CLC Genomics Workbench bioinformatics tool. Overall 37,757 out of $49,520,834$ total reads in one lymphocyte line (\# K4413-Mi) and 28,178 out of $45,335,960$ reads in the other lymphocyte line (\# K4123-Mi) were identified as EBV sequences. The two EBV genomes with estimated 35.22-fold and 31.06-fold sequence coverage respectively, designated K4413-Mi EBV and K4123-Mi EBV (GenBank accession number KC440852 and KC440851 respectively), are characteristic of type-1 EBV.

Conclusions: Sequence comparison and phylogenetic analysis among K4413-Mi EBV, K4123-Mi EBV and the EBV genomes previously reported to GenBank as well as the NA12878 EBV genome assembled from database of the 1000 Genome Project showed that these 2 EBVs are most closely related to B95-8, an EBV previously isolated from a patient with infectious mononucleosis and WT-EBV. They are less similar to EBVs associated with nasopharyngeal carcinoma (NPC) from Hong Kong and China as well as the Akata strain of a case of Burkitt's lymphoma from Japan. They are most different from type 2 EBV found in Western African Burkitt's lymphoma.
\end{abstract}

Keywords: Epstein Barr Virus, Mycoplasma, Next-generation sequencing, Human immortalized B lymphocytes

\section{Background}

Epstein-Barr virus (EBV) is a ubiquitous gamma-herpesvirus highly prevalent in all human populations. The virus is associated with both non-malignant diseases and a number of human cancers. After the first discovery of EBV particles in cultured lymphoma cells from patients with Burkitt's lymphoma (BL) [1,2], EBV and its encoded molecules were also detected in other disease processes and malignancies such as undifferentiated nasopharyngeal carcinoma (NPC)

\footnotetext{
* Correspondence: ShyhChing.Lo@fda.hhs.gov

Tissue Microbiology Laboratory, Division of Cellular and Gene Therapies, Office of Cellular, Tissue and Gene Therapy, Center for Biologics Evaluation and Research, Food and Drug Administration, NIH Building 29B, Rm. 1NN06, 29 Lincoln Dr., 20892-4555 Bethesda, MD, USA
}

[3], infectious mononucleosis [4], Hodgkin's disease [5,6], and gastric carcinoma $[7,8]$ etc.

Eight EBV whole-genome sequences have been reported from previous studies. B95-8 (GenBank accession number V01555.2), derived from a North American case of infectious mononucleosis [9], was the first completely sequenced EBV genome. A more complete $171 \mathrm{~kb}$ wild-type EBV reference genome, WT-EBV, (GenBank accession number NC_007605.1/AJ507799.2) was later constructed using B95-8 as a backbone while an $12 \mathrm{~kb}$ missing fragment in the EBV genome was provided by the EBV sequence found in Raji cells [10,11]. AG876 (DQ279927.1) from a Western African case of Burkitt's lymphoma is the only complete

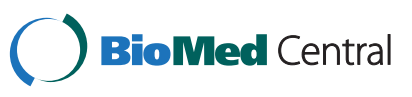


type 2 EBV sequence to date [12]; GD1 (AY961628.3), GD2 (HQ020558.1) and HKNPC1 (JQ009376.1) are all EBV genomes derived from NPC patients or NPC tumours. Specifically, GD1 was isolated from the saliva of an NPC patient, while GD2 and HKNPC1 were isolated from an NPC tumour or tumour biopsy. GD1 was sequenced using the conventional shotgun sequencing. GD2 and HKNPC1 were very recently sequenced using the next generation sequencing (NGS) Illumina platform [13-15]. Moreover, 2 EBV genomes, Akata (KC207813) and Mutu (KC207814), were reported recently from cases of Burkitt's lymphomas from Japan and Kenya, respectively, by NGS technology [16].

Our laboratory has recently established a new high throughput sequencing (HTS) capability using the MiSeq platform. Currently, we are evaluating various workflows using different programs/tools for the effective detection of a target viral genome as well as novel viral genes in computational analysis of massive amounts of sequencing data generated by NGS technology. Our objective is to effectively detect and characterize infectious viruses in human host cells or tissues intended as therapeutic biologics by using the new technology. For this purpose, we conducted an experimental genomic sequencing study of 2 human B cell lines that underwent spontaneous immortalization promoted by infection of the cell culture with mycoplasma [17]. These 2 immortalized human B lymphocyte cell lines were previously shown to be monoclonal in nature and positive for the EBV LMP1 antigen [17]. Using available computational tools, we identified and extracted EBV sequences from genomic sequencing data of the 2 B-lymphocyte cell lines, assembled them into single genomes and compared them with the sequences of all the EBV genomes previously reported to the GenBank. In addition, we identified and extracted EBV sequences from the 1000 human genomes project sequences which had been deposited in NCBI for comparison. The project has been studying human genomic DNA of human peripheral blood B lymphocytes immortalized by EBV infections in culture. The present study provided a rapid genomic comparison and characterization of EBV strains associated with different human diseases along with their pathogenesis and geographical distributions.

\section{Results}

\section{Taxonomic classification of sequences obtained from} MiSeq

DNAs were isolated from the cultures of 2 previously established, spontaneously immortalized human B lymphocyte cell lines (K4413-Mi and K4123-Mi) for genomic sequencing. A total of 49,520,834 sequencing reads of 168 -base paired-end reads were generated by the MiSeq sequencer resulting in $8.3 \mathrm{~Gb}$ of sequence data from the K4413-Mi cell line. Similarly, a total of 45,335,960 reads of 198-base paired-end reads resulting in $8.9 \mathrm{~Gb}$ of sequence data were collected from the K4123-Mi cell line. Those short reads that passed quality control filtering were first aligned to the database of human reference genome sequences (hg19, GRCh37; http://hgdownload. cse.ucsc.edu/goldenPath/hg19/bigZips/). After removing the sequencing reads that aligned with human reference genome database, the remaining reads were aligned against the bacterial, fungal, and viral nucleotide databases sequentially (ftp://ftp.ncbi.nlm.nih.gov/genomes/ Bacteria/, 04/27/2013 version; ftp://ftp.ncbi.nlm.nih.gov/ genomes/Fungi/, 03/25/2013 version; ftp://ftp.ncbi.nlm. nih.gov/genomes/Viruses/, 05/22/2013 version) to determine the sequence composition using CLC Genomic Workbench (http://www.clcbio.com/, version 6.0.2). Of the 49,520,834 sequencing reads from K4413-Mi cell line, $97.2 \%$ (48,132,751 reads), $0.011 \%$ (5,753 reads), $0.025 \%$ (12,572 reads), and $0.075 \%$ (36,904 reads) were classified as human, bacteria, fungi, and virus related sequences, respectively (Figure 1A). Of the 45,335,960 original reads from K4123-Mi cell line, 98.6\% (44,712,404 reads), $0.017 \%$ (7,759 reads), $0.019 \%$ (8,714 reads) and $0.059 \%$ (26,528 reads) were classified as human, bacteria, fungi, and virus related sequences, respectively (Figure 1C).

Of the virus-related reads identified in both cell lines, $97.6 \%$ of 36,904 reads in the K4413-Mi cell line and $98.1 \%$ of 26,528 reads in the K4123-Mi cell line were classified as EBV-specific sequences (Figure 1B, 1D). In the K4413-Mi cell line virus-related reads, there were 281 short reads classified as sequences related to Enterobacteria phage phiX174. There were 593 and 548 short reads classified as "other Herpesvirus" in K4413-Mi and K4123-Mi cell line virus-related reads, respectively (Figures 1B, D).

\section{Assembly of EBV genomes from sequences mapping with WT-EBV}

In order to assemble the K4413-Mi and K4123-Mi EBV genomes, we mapped the total sequencing reads of K4413-Mi and K4123-Mi to the reported EBV reference genome (WT-EBV, NC_007605) separately using the CLC Genomics Workbench. The mapped reads were considered to represent EBV-related sequences. Overall 37,757 out of $49,520,834$ total reads in one lymphocyte line (K4413-Mi Line) and 28,178 out of 45,335,960 total reads in the second lymphocyte line (K4123-Mi Line) were identified as EBV-related sequences. The EBV-related sequences identified in K4413-Mi cell line covered 99.99\% of the entire reference WT-EBV genome. The average coverage was 35.22 -fold. There were 10 gaps in the K4413-Mi EBV genome assembled, with gap lengths varying from $1 \mathrm{bp}$ to $105 \mathrm{bp}$. The total gap length was 352 bp. For the K4123-Mi EBV genome assembly, the EBV-related sequences identified covered $99 \%$ of the 


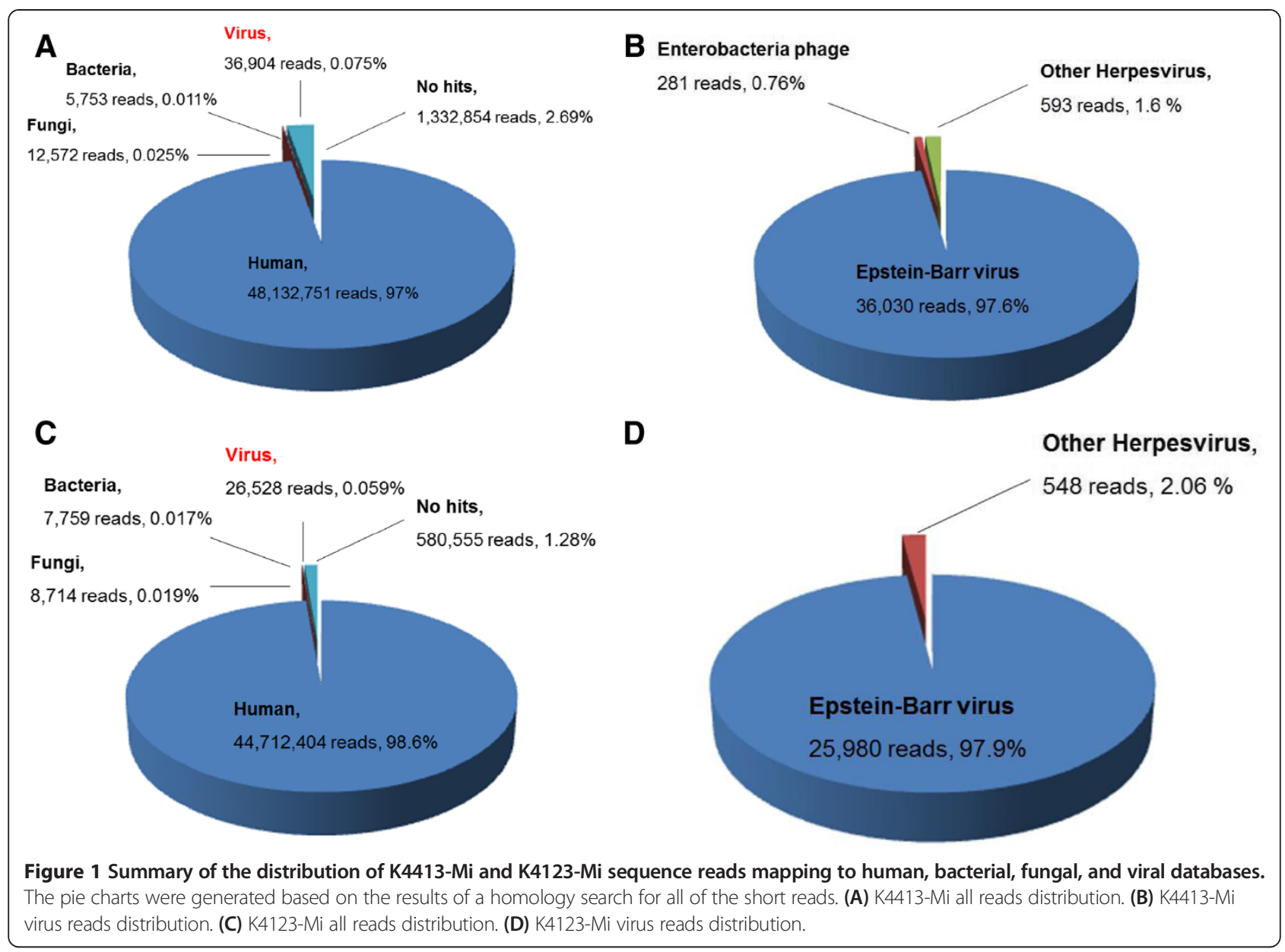

WT-EBV reference genome. The average coverage was 31.06-fold. There were 18 gaps in the K4123-Mi EBV genome assembled, and the gap length varied from $1 \mathrm{bp}$ to $177 \mathrm{bp}$. The total gap length was $993 \mathrm{bp}$. Finally, the complete genome of K4413-Mi EBV was 171,843 bp (with 352 "N"). The K4123-Mi EBV genome was 171,793 bp (with 993 "N"). Both genomes have a GC-content of 59.5\%. Both genomes were also annotated using the information derived from the reference genome of WT-EBV (NC_007605) sequence, and submitted to GenBank (GenBank accession number KC440852 and KC440851).

\section{Identification and extraction of EBV sequences from NA12878 data}

To evaluate if the bioinformatics tool used in the study could be equally effective in identifying and characterizing viral genome sequences from much more massive human genome sequencing data generated by NGS technology and to compare genome sequences of EBV identified in our "spontaneously" immortalized B cells with those in B cells transformed by acute EBV infection in culture, we downloaded sequencing raw data from NCBI of 1000 human genomes project. In the human1000 genomes project (http://www.1000genomes.org/), human genomic DNA was isolated from human B cells immortalized by EBV infection in culture [18] and deeply sequenced. The human genomic sequences obtained by this project have been deposited in NCBI. In this study we identified and extracted the EBV sequences from NA12878 human genomic sequences deposited in NCBI for comparison with EBVs in our B-cell lines. We downloaded 846,783,709 Illumina 36 bp short reads from $\mathrm{ftp} / / / \mathrm{ftp}$-trace.ncbi.nih.gov/1000genomes/ftp/data/NA12878/ sequence_read/ and used CLC Genomics Workbench software to map these reads with the WT-EBV reference genome (NC_007605). In the total reads downloaded, $2,219,104(0.26 \%)$ short reads mapped with the WT-EBV and covered $100 \%$ of the reference genome. We assembled the NA12878 EBV genome sequence from the raw reads mapping to the WT-EBV. We obtained a single contig with 171,852 bp consensus sequence from the assembly. We named the consensus sequence of the EBV genome 
NA12878 EBV. It was estimated that each immortalized human lymphocyte induced by infection of B95-8 EBV carried 102 copies of EBV genomes.

Since the large majority of the human peripheral blood $B$ lymphocytes used in the human genomes study were transformed in culture by infections of B95-8 strain of EBV, it was surprising that the segment of sequence $(\sim 12 \mathrm{~kb})$ between $139 \mathrm{~kb}$ and $151 \mathrm{~kb}$ of WT-EBV genome that B95-8 EBV genome does not possess could also be found in the assembled NA12878 EBV consensus genome sequence. Further analysis however revealed this specific $\sim 12 \mathrm{~kb}$ segment of gene sequences (marked with different colour on NA12878 EBV consensus genome in Figure 2) was being covered less than $1 / 20$ folds compared with the average 464.9-fold coverage of the rest of EBV genome. It appeared a small portion of human peripheral blood B cells transformed by B95-8 strain of EBV in 1000 human genomes project might already be infected by EBVs carrying the gene segment that was not present in B95-8 EBV. But the sequencing data clearly demonstrated the large majority of EBV genome sequences deposited in the project appeared to be closely related to the B95-B strain lacking this specific segment of genes.

\section{K4413-Mi EBV and K4123-Mi EBV are more closely related} to B95-8, WT-EBV, Mutu and NA12878 EBV

Pair-wise alignment of the newly assembled K4413-Mi EBV, K4123-Mi EBV, NA12878 EBV and 7 other EBV strains previously reported to GenBank with the WT-EBV genomic sequence was performed. Figure 2 shows the comparison result displayed by using the Mvista program (http://genome.lbl.gov/vista/mvista/submit.shtml).
Overall sequence similarities for the WT-EBV genome with the 10 other EBV genomes are high: K4413-Mi EBV (99\%), K4123-Mi EBV (99\%), NA12878 EBV (100\%), B95-8 (95\%), Akata (98\%), Mutu (100\%), GD1 (97\%), GD2 (96\%), HKNPC (96\%), AG876 (96\%). The region (13 kb - $42 \mathrm{~kb})$ with highly repetitive sequence in the EBV genome where majority of gaps and ambiguity reads occurred was not included for the genomic comparison (Figure 2). Figure 2 shows that sequences in OriP (origin of DNA replication), IR 3, 4 (internal repetitive family), and TR (terminal repetitive family) regions among the EBV genomes have lower similarity. There were also many sequence variations in polymorphic genes, such as EBNA-3A, 3B, and 3C among these EBV genomes (Figure 2).

More importantly, comparison of sequences in the region spanning EBNA3A, $3 \mathrm{~B}$ and $3 \mathrm{C}$ for $\mathrm{K} 4413-\mathrm{Mi}$ EBV and K4123-Mi EBV against WT-EBV genome revealed much higher similarity with those of the B95-8 and Mutu genomes, but very different from the sequence in this region of the AG876 genome.

\section{Phylogenetic comparison for the genomic sequences of K4413-Mi and K4123-Mi EBV with other EBVs asso- ciated with various human diseases and from different geographic areas}

Multiple sequence alignment of K4413-Mi EBV, K4123$\mathrm{Mi} \mathrm{EBV}$, and the other $7 \mathrm{EBV}$ genome sequences was performed using the Kalign program in EBI (http://www.ebi. ac.uk/Tools/msa/kalign/) and the Neighbour-joining trees were constructed using MEGA 5 software (http://www. megasoftware.net/) (Figure 3A). Phylogenetic analysis revealed that K4413-Mi EBV and K4123-Mi EBV were

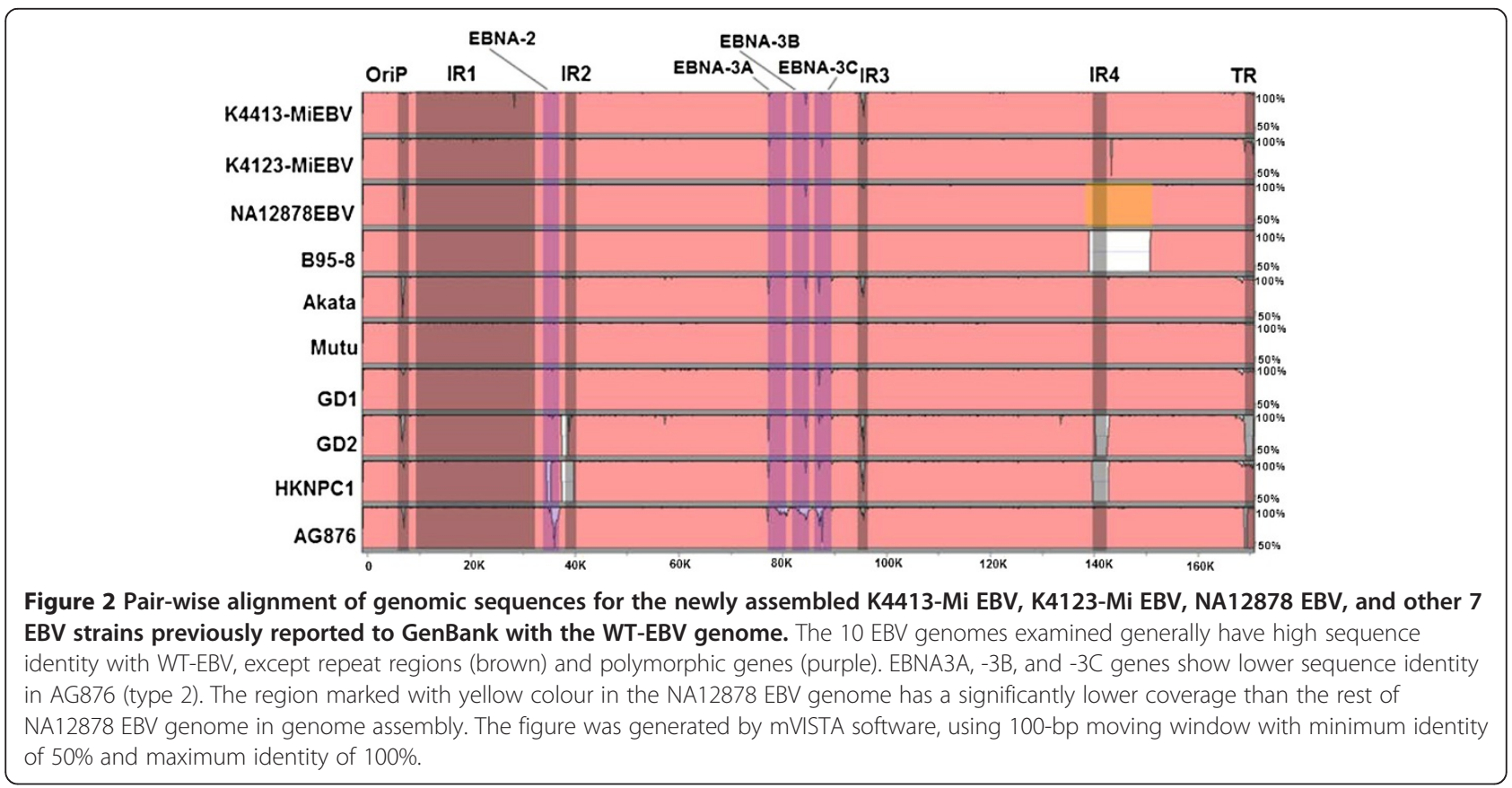




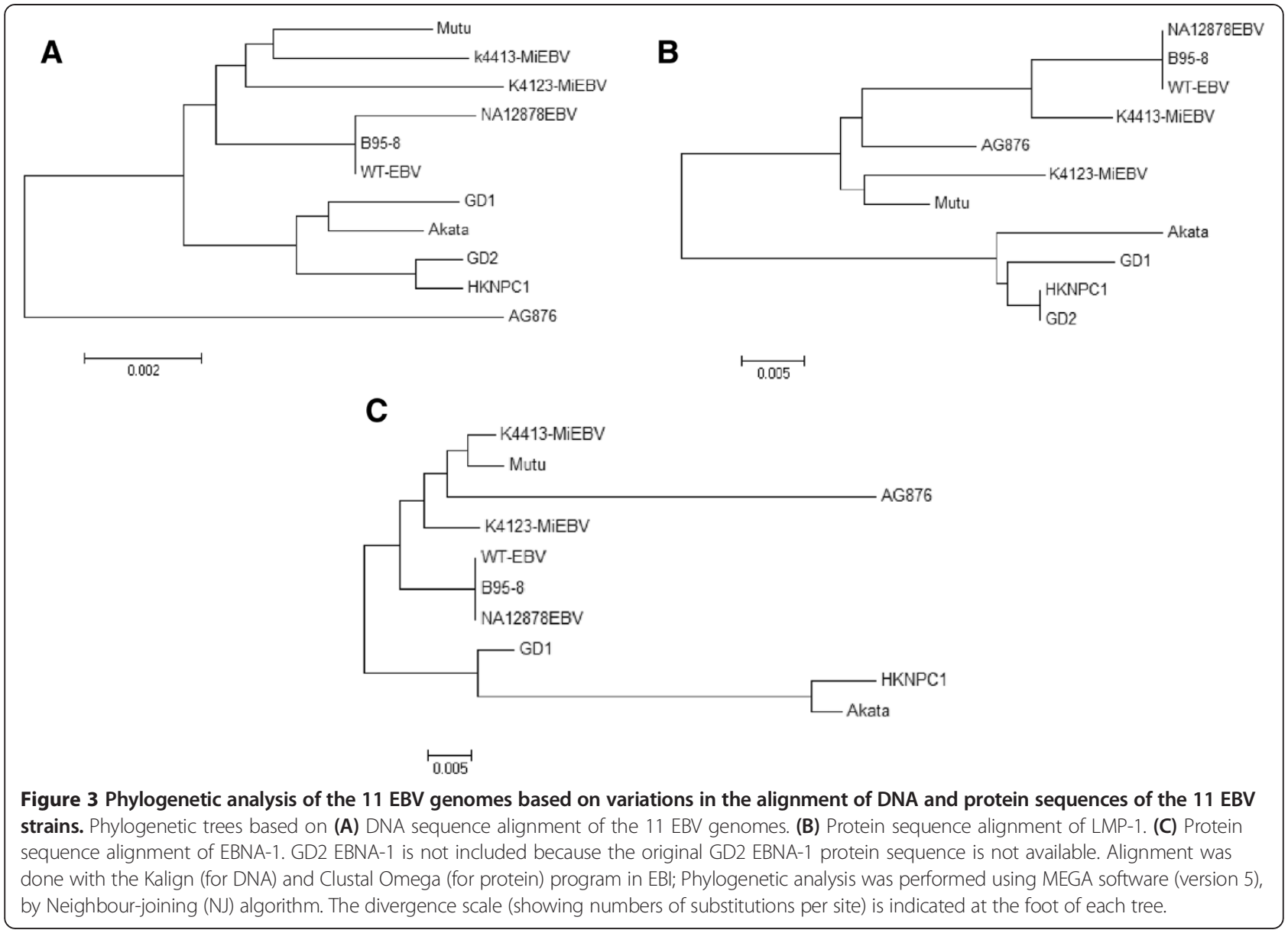

more closely related to B95-8, WT-EBV and NA12878 EBV. They were more different from GD1, GD2, and HKNPC1, EBVs associated with NPC found in China and Hong Kong as well as Akata, EBV from a case of Burkitt's lymphoma found in Japan. They were most different from AG876, the type 2 EBV found in a case of the Western African Burkitt's lymphoma. Interestingly, Mutu EBV from a case of Burkitt's lymphoma in Kenya is found to be more closely related to K4413-Mi EBV, K4123-Mi EBV and B95-8 and WT-EBV (Figure 3A). Phylogenetic trees generated from the alignment of translated amino acid sequences of LMP1 and EBNA-1 also showed that K4413-Mi EBV and K4123-Mi EBV are more distant from the NPC associated EBVs and Akata strain of EBV from Burkitt's lymphoma in East Asia (Figures 3B, C).

\section{Identification of variations from 2 immortalized human B cell lines}

We used the CLC Genomics Workbench to identify variations, including single-nucleotide variants (SNVs), indels (insertion and deletions) in K4413-Mi and K4123Mi EBVs. In comparison with the WT-EBV reference genome, a total of 444 variants with genome-wide distribution were found in the K4413-Mi EBV genomes (Figure 4B). Among them, 418 variants were homogenous SNVs; 10 variants were heterogeneous SNVs. In addition, there were 9 insertions and 7 deletions. There were 424 variants located in the coding regions and 138 variants were non-synonymous substitutions (Table 1 and Additional file 1: Table S1). In comparison with the WT-EBV reference genome, a total of 333 variants with similar genome-wide distribution were found in the K4123-Mi EBV genome (Figure 4A). Among them, 324 variants were homogenous SNVs; 1 variant was heterogeneous SNV. In addition, there were 2 insertions and 6 deletions. Among these variants, 329 variants were located in the coding region and 110 variants were nonsynonymous substitutions (Table 1 and Additional file 2: Table S2).

It is important to note that K4413-Mi and K4123-Mi shared 97 common variants (Figure 4C). The common 


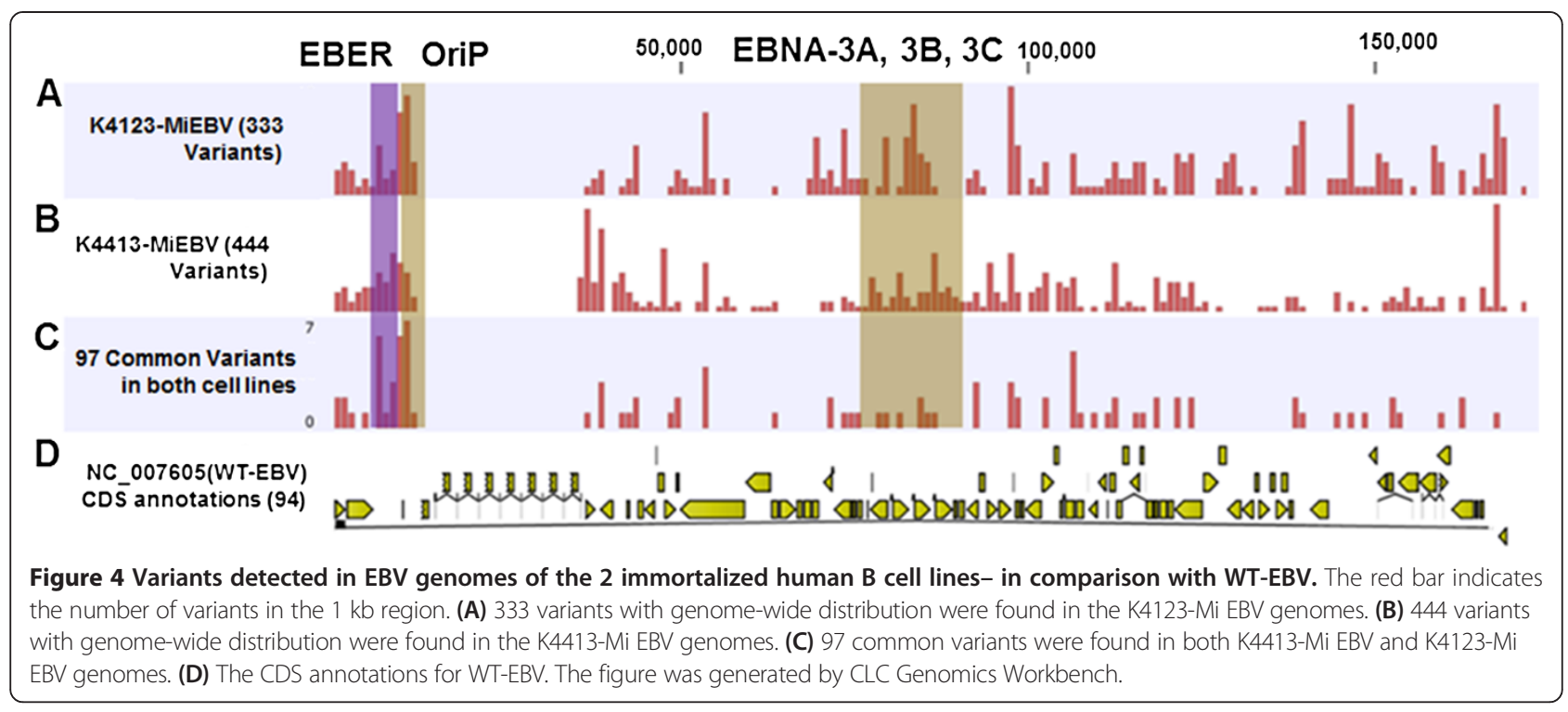

variants were apparently clustered in certain specific regions, e.g., from $6 \mathrm{~kb}$ to $10 \mathrm{~kb}$, which is the region that encodes EBER-1, 2 (EBV-encoded RNA) and contains the OriP (the latent cycle origin of DNA replication), and from $79 \mathrm{~kb}$ to $83 \mathrm{~kb}$, the region that encodes EBNA3 etc (Figure 4).

\section{Discussion}

We are evaluating the capability of detecting/characterizing infectious viruses in human host cells through HTS genomic sequencing using the Illumina Miseq platform and different computational tools. In this study, we examined 2 human B lymphocyte cell lines that underwent spontaneous immortalization promoted by mycoplasma infection of the cell culture using the Illumina MiSeq platform. We used the HTS data analytic program, CLC Genomics Workbench, to classify the massive short sequence reads (49.5 and 45.3 million reads, $\sim 175$ bp/each) generated from genomic sequencing of the 2 cell lines by

Table 1 Summary of variations identified in EBVs of the K4413-Mi and K4123-Mi cell lines-in comparison with WT-EBV

\begin{tabular}{lll}
\hline & K4413-Mi EBV & K4123-Mi EBV \\
\hline No. of variations & 444 & 333 \\
No. of SNVs (single-nucleotide variants) & 428 & 325 \\
No. of homozygous SNVs & 418 & 324 \\
No. of heterozygous SNVs & 10 & 1 \\
No. of indels (insertion or deletion) & 16 & 8 \\
No. of coding regions change & 424 & 329 \\
No. of non-synonymous substitutions & 138 & 110 \\
\hline
\end{tabular}

mapping these sequences with human, bacterial, fungal, and viral genomic databases from NCBI database respectively (Figure 1A-D). The majority of reads obtained (97.2\% or $8.1 \mathrm{~Gb}$ for K4413-Mi cell line and $98.6 \%$ or $8.8 \mathrm{~Gb}$ for $\mathrm{K} 4123-\mathrm{Mi}$ cell line), that was classified as human sequences, had approximately 1.22 -fold and 1.34 -fold coverage of a diploid human genome $(6.6 \mathrm{~Gb})$.

Consensus EBV genomes were constructed according to the mapping result of the raw read with WT-EBV by using the CLC Genomics Workbench. The K4413-Mi EBV consensus genome was assembled by using 37,757 EBV-related reads (0.075\% of total sequencing raw reads), had $171,843 \mathrm{bp}$ in length with $~ 35.22$-fold coverage of EBV genome. Thus, it is estimated that there are $\sim 29$ copies (35.22 vs 1.22 coverage) of EBV genome in a single cell of the K4413-Mi lymphocyte cell line. Similarly, the K4123-Mi EBV genome was assembled by using 28,178 EBV-related reads, and was 171,793 bp in length with $\sim 31.06$-fold coverage of the EBV genome. It is estimated that there are $\sim 23$ copies (31.06 vs 1.34 coverage) of the EBV genome in a single cell of the K4123-Mi lymphocyte cell line. The genome copy numbers of EBV found in these resting human B lymphocytes that underwent spontaneous immortalization are apparently higher than those previously reported in the undifferentiated NPC tumours. In GD2, there were $\sim 6$ copies of EBV genome found in a single NPC tumour cell [14]. In comparison, the NA12878 EBV genome constructed from NCBI database revealed an average of 102 copies of EBV genome in each immortalized human lymphocyte induced by infection of B95-8 EBV in culture. It appears that there are many more copies of EBV genomes that are present in each transformed human B lymphocyte 
induced by acute B95-8 EBV infection than in spontaneously transformed EBV-positive B-cell promoted by infections of mycoplasma. The copy number of EBV genomes found in an NPC tumour cell is evidently the lowest.

Analysis of our results show that some sequences, albeit very few, obtained in the genomic sequencing of K4413-Mi and K4123-Mi human lymphocyte cell lines mapped to bacteria, fungi, and non-EBV viruses (Figure 1A-D). None-biased parallel HTS is capable of picking up trace amounts of DNA molecules present in culture media and serum. Most of the bacteria-related sequences were mapped to Enterobacteria. When matched with the NCBI viral database using the CLC Genomics Workbench, 281 reads in K4413-Mi cell line were found to be Enterobacteria phage phiX174. It is possible that the bacteria in the medium also carried the Enterobacteria phage phiX174. Consistent with the original study finding [17], no mycoplasma sequence was identified in these cell lines. There are 593 reads in K4413-Mi cell line and 548 reads in K4123-Mi were classified to be other Herpesvirus-related sequence, not the EBV virus (Figure 1B, D). We conducted the individual BlastN for these 1141 reads. Most of these reads also matched with EBV virus with low homology, and some reads matched with Human sequence. In this context, 2.69\% and 1.28\% of reads showed "no hits" in mapping against the 4 genomic databases (human, bacteria, fungi, and virus) for the K4413-Mi and K4123-Mi cell lines, respectively. When these sequences were mapped using BlastN against NCBI non-redundant databases, most of these sequences were found to be human sequences. The CLC Genomics Workbench is a very powerful tool for NGS data analysis. It allows us to quickly identify the read composition from the massive amount of data. We also can effectively detect and assemble the target viral EBV genome with the CLC Genomics Workbench. Of course, all software programs have its advantages and drawbacks. For bioinformatics tools, parameter settings and databases used will affect the outcome of the analysis. Different bioinformatics tools are likely to produce different results. We also tried the DNASTAR, SOAP package, BWA and other bioinformatics tools for our analysis. The results are slightly different from using the CLC Genomics Workbench (data not shown). In this study, we only present our analysis with the CLC program.

Whole-genome sequencing of EBV in the infected cells enabled the determination and thus comparison of EBV variations at the genome level. The constructed genomes of K4413-Mi EBV and K4123-Mi EBV are highly similar to each other and to the 8 other reported EBV genomes in the GenBank. However, there are apparent degrees of variations among the genomes of these EBVs studied. Phylogenetic comparison of these EBV genomes revealed that K4413-Mi EBV and K4123-Mi EBV are more closely related to B95-8, EBV isolated from a patient with infectious mononucleosis. They are evidently more distant from GD1, GD2, and HKNPC1, EBVs associated with NPC tumours. Specific comparison for the two particular EBV genes (LMP1 and EBNA-1) that were considered risk-loci in NPC [19] and commonly used for classification also revealed that K4413-Mi EBV and K4123-Mi EBV are closer to B95-8 and more different from NPC-related EBVs (Figure 3B, C). Furthermore, both K4413-Mi EBV and K4123-Mi EBV lacked the 30 bp deletion at the carboxyl terminus and a specific amino acid substitution (Asp) at codon 335 with reference to Gly in B95-8 LMP1, a feature that was reportedly present in over $90 \%$ of EBVs found in NPC biopsies [20]. Moreover, K4413-Mi EBV and K4123-Mi EBV were evidently more distant from AG876, EBV isolated from African Burkitt's lymphoma (Figures 2, 3A). Specific comparison of sequences in the EBNA3 region that had been used for classification of different EBV subtypes [21], similarly revealed K4413-Mi EBV and K4123-Mi EBV were more closely related to B95-8, a subtype 1 EBV (Figure 2). They were most distant from AG876, a subtype 2 EBV from the Western African case of Burkitt's lymphoma.

Inclusion of the 2 most recently reported sequences of EBV genomes, Akata and Mutu, in our analysis of genomic sequence variations reveals the significant geographical distribution factor in addition to the factors of disease or tissue association. K4413-Mi EBV and K4123-Mi EBV are clearly more closely related to B95-8 strain and WT-EBV and more different from GD1, GD2 and HKNPC1 EBVs associated with NPC in the East Asia and Akata-EBV strain from a Japanese case of Burkitt's lymphoma. They are clearly most different from the EBV of Western Africa case of Burkitt's lymphoma. However, it is interesting to find that Mutu, EBV strain from a Kenya case of Burkitt's lymphoma in the East Africa is more closely related to K4413-Mi EBV, K4123-Mi EBV, B95-8 and WT-EBV found in the North America (Figure 3A). More genome sequencing data of EBVs from different geographic regions in the world in the future could provide important information of the history or the route of EBV dissemination as well as its evolution. In this study, the geographical distribution factor in sequence variations among these EBVs can similarly be observed from the alignments of translated amino acid sequences of LMP1 and EBNA-1 genes among these EBVs of different origins (Figures 3B, C).

It may also be important to note that there were 10 heterogeneous SNPs found in the K4413-Mi EBV genome. There was also 1 heterogeneous SNPs found in the K4123-Mi EBV genome. The finding would suggest that there could be more than two EBV variants or quasi species within the K4413-Mi cell and K4123-Mi cell. 


\section{Conclusions}

We used the Illumina MiSeq sequencing platform, a NGS technology and convenient computational tools, CLC Genomics Workbench, to identify and extract EBV sequences from human B lymphocyte cell lines that underwent spontaneous immortalization promoted by infection of mycoplasma in culture. Genomes of EBVs identified in the 2 immortalized human B lymphocytes were constructed and compared with all the other EBV genomes available in GenBank. The study has demonstrated how viral agents in infected human cells could be rapidly identified, characterized and compared at the genomic level through genomic sequencing and effective computational analysis. The EBVs found in these human B lymphocytes are most closely related to B95-8 and WTEBV. They are less similar to EBVs found in NPC and Burkitt's lymphoma found in the East Asia. They are most different from EBV associated with the Western African Burkitt's lymphoma based on whole genome sequences.

\section{Methods}

Sample preparation and short-read DNA sequencing

Two EBV-positive human B cell lines that underwent spontaneous immortalization promoted by infections of mycoplasma (namely K4413-Mi and K4123-Mi) were cultured as described [17]. Both of the B-cell lines were established from buffy coats of healthy blood donors obtained from Department of Transfusion Medicine, Warren Grant Magnuson Clinical Center, National Institutes of Health (Bethesda, MD). Personal information concerning these samples had been recorded in such a manner that subjects could not be identified, directly or through identifiers according to the HIPAA rules. Thus, the ethnic and geographic origins of the donors are not available. Genomic DNAs from these cell lines were extracted using the DNAse Blood and Tissue Kit (Qiagen). 50 ng DNA from each cell line was subjected to DNA library construction using the Nextera DNA Kit (Illumina) according to the manufacturer's protocol. Briefly, DNA library was prepared through procedures that include tagmentation and 5- cycle PCR amplification. The DNA library has insert sizes ranging from $250 \mathrm{bp}$ to $1000 \mathrm{bp}$. Two sequencing runs were conducted for each sample studied. The 1st run sequencing used Illumina Miseq reagent kit (300 cycles for the $2 \times 150$ bp pair-end sequencing). The 2 nd run of sequencing used Illumina Miseq reagent kit V2 (500 cycles for the $2 \times 250$ bp pairend sequencing). The reads that passed Illumina quality control filtering were used as raw data for further bioinformatics analysis.

\section{Taxonomic analysis of the short sequencing reads}

The reference human database (hg19 human reference assembly) was downloaded from the University of California,
Santa Cruz (UCSC), (http://hgdownload.cse.ucsc.edu/down loads.html\#human); bacterial, fungal and viral databases were downloaded from National Center for Biotechnology Information (NCBI) (ftp://ftp.ncbi.nlm.nih.gov/genomes/ Bacteria/, 04/27/2013 version; ftp://ftp.ncbi.nlm.nih.gov/ genomes/Fungi/, 03/25/2013 version; ftp://ftp.ncbi.nlm.nih. gov/genomes/Viruses/, 05/22/2013 version). These 4 databases were uploaded to the CLC Genomics Workbench to build the local database. All sequencing reads were first aligned to the hg19 human database using the CLC Genomics Workbench with the default parameters (http://www.clcbio.com/, version 6.0.2). The matched human reads were removed from the raw data set. Likewise, the remaining reads were aligned to the locally built bacterial, fungal and viral databases sequentially using the CLC Genomics Workbench.

Assembly of the K4413-Mi EBV and K4123-Mi EBV genomes The total raw reads of the K4413-Mi or K4123-Mi cell line were aligned to the WT-EBV by using the CLC Genomics Workbench with the default parameters. The matched reads for each cell line were used for the EBV genome assembly.

\section{Identification of variations in the EBV genomic sequences} from the K4413-Mi and K4123-Mi cell lines

Using WT-EBV as a reference, the single nucleotide variations (SNVs), inserts and deletions (indels) and multiple nucleotide variations (MNVs) were identified from the K4413-Mi and K4123-Mi cell lines. MiSeq reads by using the CLC Genomics Workbench with the default parameters.

\section{Comparative and phylogenetic analysis}

A global comparison and visualization of WT-EBV against K4413-Mi EBV, K4123-Mi EBV, NA12878 EBV, B95-8, AG876, Akata, Mutu, GD1, GD2 and HKNPC1 was performed using the mVISTA program (http://genome.lbl.gov/ vista/mvista/submit.shtml) with 100-bp moving window.

The global sequence alignments of the 11 EBV genomes and individual gene sequence alignments were analyzed using Kalign and Clustal Omega in EBI (http://www.ebi. ac.uk/Tools/msa/kalign/; http://www.ebi.ac.uk/Tools/msa/ clustalo/) respectively. The alignments were used to generate phylogenetic trees using Molecular Evolutionary Genetics Analysis (MEGA) software, version 5.0 [22] with Neighbour-joining (NJ) algorithm.

\section{Nucleic sequence accession numbers}

The full-length sequences of K4413-Mi EBV and K4123-Mi EBV were submitted to the GenBank database with the accession number KC440852 and KC440851. 


\section{Additional files}

Additional file 1: Table S1. Variants identified in K4413-Mi EBV in comparison with WT-EBV.

Additional file 2: Table S2. Variants identified in K4123-Mi EBV in comparison with WT-EBV.

\section{Competing interests}

The authors declare that they have no competing interests.

\section{Authors' contributions}

HYL and SCL conceived and designed the experiments. ST and BJL performed cell culture study. TWL and HYL conducted genomic sequencing. HYL and GCH analyzed the sequencing data. SCL oversaw the progress of project and reviewed all the study data. $\mathrm{HYL}$ and $\mathrm{SCL}$ wrote the original draft of the manuscript. TWL, GCH, BJL and ST contributed to the discussion and writing of the final version of the manuscript. All authors reviewed and approved the final manuscript.

\section{Acknowledgements}

We thank Cindy Clark from the NIH library for critically editing the manuscript; CLC Genomics Workbench technical support and NIH Helix technical support are acknowledged for their technical assistance with the CLC Genomics Workbench and NIH supercomputer, respectively. Dr. Jing Han and Dr. Jennifer Catalano conducted constructive reviews of the paper.

\section{Funding sources}

The study was supported in part by FDA Modernizing Science research grant and supported in part by an appointment to the Research Participation Program at the CBER of FDA administered by the Oak Ridge Institute for Science and Education through an interagency agreement between the U.S. Department of Energy and the U.S. Food and Drug Administration.

Received: 16 January 2013 Accepted: 12 November 2013 Published: 19 November 2013

\section{References}

1. Epstein MA, Achong BG, Barr YM: Virus particles in cultured lymphoblasts from Burkitt's lymphoma. Lancet 1964, 1:702-703.

2. zur Hausen $H$, Schulte-Holthausen $H$, Klein G, Henle W, Henle G, Clifford P, et al: EBV DNA in biopsies of Burkitt tumours and anaplastic carcinomas of the nasopharynx. Nature 1970, 228:1056-1058.

3. Wolf $\mathrm{H}$, zur Hausen $\mathrm{H}$, Becker $\mathrm{V}$ : EB viral genomes in epithelial nasopharyngeal carcinoma cells. Nat New Biol 1973, 244:245-247.

4. Henle G, Henle W, Diehl V: Relation of Burkitt's tumor-associated herpes-ytpe virus to infectious mononucleosis. Proc Natl Acad Sci USA 1968, 59:94-101.

5. Kapatai G, Murray P: Contribution of the Epstein Barr virus to the molecular pathogenesis of Hodgkin lymphoma. J Clin Pathol 2007, 60:1342-1349.

6. Weiss LM, Movahed LA, Warnke RA, Sklar J: Detection of Epstein-Barr viral genomes in reed-sternberg cells of Hodgkin's disease. N Engl J Med 1989, 320:502-506.

7. lizasa H, Nanbo A, Nishikawa J, Jinushi M, Yoshiyama H: Epstein-Barr Virus (EBV)-associated gastric carcinoma. Viruses 2012, 4:3420-3439.

8. Imai S, Koizumi S, Sugiura M, Tokunaga M, Uemura Y, Yamamoto N, et al: Gastric carcinoma: monoclonal epithelial malignant cells expressing Epstein-Barr virus latent infection protein. Proc Natl Acad Sci USA 1994, 91:9131-9135.

9. Miller G, Lipman M: Release of infectious Epstein-Barr virus by transformed marmoset leukocytes. Proc Natl Acad Sci USA 1973, 70:190-194.

10. Baer R, Bankier AT, Biggin MD, Deininger PL, Farrell PJ, Gibson TJ, et al: DNA sequence and expression of the B95-8 Epstein-Barr virus genome. Nature 1984, 310:207-211.

11. de Jesus O, Smith PR, Spender LC, Elgueta KC, Niller HH, Huang D, et al: Updated Epstein-Barr virus (EBV) DNA sequence and analysis of a promoter for the BART (CST, BARF0) RNAs of EBV. J Gen Virol 2003, 84:1443-1450.

12. Dolan A, Addison C, Gatherer D, Davison AJ, McGeoch DJ: The genome of Epstein-Barr virus type 2 strain AG876. Virology 2006, 350:164-170.

13. Kwok H, Tong AH, Lin CH, Lok S, Farrell PJ, Kwong DL, et al: Genomic sequencing and comparative analysis of Epstein-Barr virus genome isolated from primary nasopharyngeal carcinoma biopsy. PLOS One 2012, 7:e36939.
14. Liu P, Fang X, Feng Z, Guo YM, Peng RJ, Liu T, et al: Direct sequencing and characterization of a clinical isolate of Epstein-Barr virus from nasopharyngeal carcinoma tissue by using next-generation sequencing technology. J Virol 2011, 85:11291-11299.

15. Zeng MS, Li DJ, Liu QL, Song LB, Li MZ, Zhang RH, et al: Genomic sequence analysis of Epstein-Barr virus strain GD1 from a nasopharyngeal carcinoma patient. J Virol 2005, 79:15323-15330.

16. Lin Z, Wang X, Strong MJ, Concha M, Baddoo M, Xu G, et al: Whole-genome sequencing of the Akata and Mutu Epstein-Barr virus strains. J Virol 2013, 87:1172-1182.

17. Zhang S, Tsai S, Wu TT, Li B, Shih JW, Lo SC: Mycoplasma fermentans infection promotes immortalization of human peripheral blood mononuclear cells in culture. Blood 2004, 104:4252-4259.

18. 1000 Genomes Project Consortium, Abecasis GR, Altshuler D, Auton A, Brooks LD, Durbin RM, Gibbs RA, Hurles ME, McVean GA: A map of human genome variation from population-scale sequencing. Nature 2010 467:1061-1073.

19. Zhang XS, Wang HH, Hu LF, Li A, Zhang RH, Mai HQ, et al: V-val subtype of Epstein-Barr virus nuclear antigen 1 preferentially exists in biopsies of nasopharyngeal carcinoma. Cancer Lett 2004, 211:11-18.

20. Zhang XS, Song KH, Mai HQ, Jia WH, Feng BJ, Xia JC, et al: The 30-bp deletion variant: a polymorphism of latent membrane protein 1 prevalent in endemic and non-endemic areas of nasopharyngeal carcinomas in China. Cancer Lett 2002, 176:65-73.

21. Sample J, Young L, Martin B, Chatman T, Kieff E, Rickinson A, et al: Epstein-Barr virus types 1 and 2 differ in their EBNA-3A, EBNA-3B, and EBNA-3C genes. J Virol 1990, 64:4084-4092.

22. Tamura K, Peterson D, Peterson N, Stecher G, Nei M, Kumar S: MEGA5: molecular evolutionary genetics analysis using maximum likelihood, evolutionary distance, and maximum parsimony methods. Mol Biol Evol 2011, 28:2731-2739.

doi:10.1186/1471-2164-14-804

Cite this article as: Lei et al:: Identification and characterization of EBV genomes in spontaneously immortalized human peripheral blood B lymphocytes by NGS technology. BMC Genomics 2013 14:804

\section{Submit your next manuscript to BioMed Central and take full advantage of:}

- Convenient online submission

- Thorough peer review

- No space constraints or color figure charges

- Immediate publication on acceptance

- Inclusion in PubMed, CAS, Scopus and Google Scholar

- Research which is freely available for redistribution
C Biomed Central 